\title{
A CHARACTERIZATION OF FUZZY NEIGHBORHOOD COMMUTATIVE DIVISION RINGS
}

\section{T.M.G. AHSANULLAH and FAWZI AL-THUKAIR}

\author{
Department of Mathematics, College of Science \\ King Saud University \\ P.O. Box 2455, Riyadh-11451 \\ Kingdom of Saudi Arabia
}

(Received July 7, 1992 and in revised form November 20, 1992)

\begin{abstract}
We give a characterization of fuzzy neighborhood commutative division ring; and present an alternative formulation of boundedness introduced in fuzzy neighborhood rings. The notion of $\beta$-restricted fuzzy set is considered.
\end{abstract}

KEY WORDS AND PHRASES. Fuzzy neighborhood system; fuzzy neighborhood commutative division ring (FNCDR); bounded fuzzy set; $\beta$-restricted fuzzy set.

1992 AMS SUBJECT CLASSIFICATION CODES. Primary, 54A40; Secondary, 16W80.

\section{INTRODUCTION.}

The notions of fuzzy neighborhood division ring and fuzzy neighborhood commutative division ring are announced in [1] without producing any characterization theorem on the topics. In this article, our aim is to provide with such a characterization theorem.

Fuzzy neighborhood rings are studied in [2] where the concept of bounded fuzzy set is introduced. We give here an alternative equivalent formulation of boundedness in case of commutative division rings. Finally, we propose a notion of $\beta$-restricted fuzzy set where $0<\beta \leq 1$, an analouge of restricted set in topological commutative division rings.

\section{PRELIMINARIES.}

Like recent works, for instance ([1], [2], [3], [4] and [5]) the key item of this article is the notion of fuzzy neighborhood system originated by R. Lowen [6]. For our convenience, we quote below a few known definitions and useful results.

Throughout the text, we consider the triplet $(D,+, \cdot)$ either a ring, division ring or commutative division ring (whichever we require), while $D^{*}:=D \backslash\{0\}$ stands for multiplicative group of nonzero elements of commutative division ring $D$ and $D^{+}$is the additive group of $D$.

As usual, $\left.\left.I_{0}:=\right] 0,1\right]$, and $I:=[0,1]$ the unit interval. $\square$ denotes the completion of the proof. For any fuzzy set $\mu \epsilon I^{D}(=\{\mu: D \rightarrow I\}) \mu \sim$ is defined as

$$
\mu \sim(x):=\mu\left(x^{-1}\right) \forall x \in D^{*}
$$

If $x \in D$ then,

$$
x \oplus \mu(y):=1_{\{x\}} \oplus \mu(y)=\mu(y-x) \quad \forall y \varepsilon D
$$

where $1_{\{x\}}$ denotes the characteristic function of the singleton set $\{x\}$, while for any $\mu_{,} \nu_{1}, \nu_{2} \in I^{D}$ and $x \epsilon D^{*}$ 
$x \odot \mu, \nu_{1} \oplus \nu_{2}$ and $\nu_{1} \odot \nu_{2}$ are defined successively,

$$
\begin{aligned}
& x \odot \mu(y):=1_{\{x\}} \odot \mu(y)=\mu\left(y x^{-1}\right) \\
& \nu_{1} \oplus \nu_{2}(y):=\underset{s+t=y}{\vee} \nu_{1}(s) \wedge \nu_{2}(t) \\
& \text { and } \nu_{1} \odot \nu_{2}(y):={ }_{s . t} \vee=y \nu_{1}(s) \wedge \nu_{2}(t)
\end{aligned}
$$

for all $y \in D$.

Also, we define $\mu / \nu$ as

$$
\nu / \nu:=\mu \odot \nu \sim
$$

and so $1 /(1 \oplus \nu)$ is written as

$$
1 /(1 \oplus \nu)(x):=(1 \oplus \nu) \sim(x):=(1 \oplus \nu)\left(x^{-1}\right) \forall x \in D^{*} .
$$

We call $\mu$ is symmetric if and only if

$$
\mu=\sim \mu, \text { where } \sim \mu(x)=\mu(-x) \forall x \in D .
$$

The constant fuzzy set of $D$ with value $\delta \epsilon I$ is given by the symbol $\underline{\underline{\delta}}\left(\epsilon I^{D}\right)$.

We recall the so-called saturation operator $[6,7]$ which is defined on a prefilter base $F \subset I^{D}$ by

$$
\widetilde{F}=\left\{\nu \in I^{D}: \forall \delta \epsilon I_{0} \exists \nu_{\delta} \epsilon F \ni \nu_{\delta}-\delta \leq \nu\right\} .
$$

If $\Sigma:=(\Sigma(x))_{x \epsilon D}$ is a fuzzy neighborhood system on a set $D$ then $t(\Sigma)$ is the fuzzy neighborhood topology on $D$, and the pair $(D, t(\Sigma))$ is known as fuzzy neighborhood space [6].

PROPOSITION 2.1. If $(D, t(\Sigma))$ and $\left(D^{\prime}, t\left(\Sigma^{\prime}\right)\right)$ are fuzzy neighborhood spaces and $f: D \rightarrow D^{\prime}$,

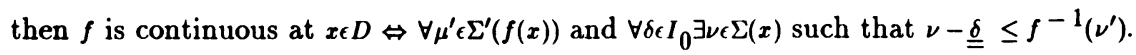

DEFINITION 2.2. Let $(D,+, \cdot)$ be a ring and $\Sigma$ a fuzzy neighborhood system on $D$. Then the quadruple $(D,+, \cdot, t(\Sigma))$ is said to be a fuzzy neighborhood ring if and only if the following are satisfied:

(FR1) The mapping $h:(D \times D, t(\Sigma) \times t(\Sigma)) \rightarrow(D, t(\Sigma)),(x, y) \mapsto x+y$ is continuous.

(FR2) The mapping $k:(D, t(\Sigma)) \rightarrow(D, t(\Sigma)), x \mapsto-x$ is continuous.

(FR3) The mapping $m:(D \times D, t(\Sigma) \times t(\Sigma)) \rightarrow(D, t(\Sigma)),(x, y) \mapsto x y$ is continuous.

PROPOSITION 2.3. Let $(D,+, \cdot t(\Sigma))$ be a fuzzy neighborhood ring and $x \in D$.

Then

(a) The left homothety $\ell_{x}:(D, t(\Sigma)) \rightarrow(D, t(\Sigma)) y \mapsto x y$ (resp. right homothety $\mathscr{x}_{x}:(D, t(\Sigma)$ ) $\rightarrow(D, t(\Sigma)), y \mapsto y x)$ is continuous. If $x$ is a unit element of $D$ then each homothety is a homeomorphism.

(b) The translation $T_{x}:(D, t(\Sigma)) \rightarrow(D, t(\Sigma)), y \mapsto y+x$, and the inversion $k$ are homeomorphisms.

(c) $\nu \epsilon \Sigma(0) \Leftrightarrow x \oplus \nu \epsilon \Sigma(x)$, i.e., $T_{x}(\nu) \epsilon \Sigma(x)$.

(d) $\nu \epsilon \Sigma(x) \Leftrightarrow-x \oplus \nu \epsilon \Sigma(0)$, i.e., $T-x(\nu) \epsilon \Sigma(0)$.

DEFINITION 2.4. Let $(D,+, \cdot)$ be a division ring, and $\Sigma$ a fuzzy neighborhood system on $D$. Then the quadruple $(D,+, \cdot, t(\Sigma))$ is said to be a fuzzy neighborhood division ring if and only if the following are true: 
(FD1) $(D,+, \cdot, t(\Sigma))$ is a fuzzy neighborhood ring.

(FD2) The mapping $r:\left(D^{*}, t\left(\Sigma_{\mid} D^{*}\right)\right) \rightarrow\left(D^{*}, t\left(\Sigma_{\mid} D^{*}\right)\right), x \mapsto x^{-1}$ is continuous, where $\Sigma_{\mid} D^{*}$ is the fuzzy neighborhood system on $D^{*}$ induced by $D$.

THEOREM 2.5. Let $(D,+, \cdot)$ be a ring and $\Sigma$ a fuzzy neighborhood system on $D$. Then the quadruple $(D,+, \cdot t(\Sigma))$ is a fuzzy neighborhood ring if and only if the following are satisfied:

(1) $\forall x \in D: \Sigma(x)=\left\{T_{x}(\nu): \nu \in \Sigma(0)\right\}$

(2) $\forall x_{0} \epsilon D, \forall \mu \epsilon \Sigma(0), \forall \delta \epsilon I_{0} \exists \nu \epsilon \Sigma(0) \ni x_{0} \odot \nu \leq \mu+\underline{\underline{\delta}}$, and $\nu \odot x_{0} \leq \mu+\delta$, i.e., the mapping $y n \rightarrow x_{0} y$ and $y \mapsto y x_{0}$ are continuous at 0 .

(3) $\forall \mu \epsilon \Sigma(0), \forall \delta \epsilon I_{0} \exists \nu \epsilon \Sigma(0) \ni \nu \oplus \nu \leq \mu+\underline{\underline{\delta}}$, i.e., the mapping $(x, y) \mapsto x+y$ is continuous at $(0,0)$.

(4) $\forall \mu \epsilon \Sigma(0), \forall \delta \epsilon I_{0} \exists \nu \epsilon \Sigma(0) \ni \nu \leq \sim \mu+\underline{\underline{\delta}}$, i.e., the mapping $x \mapsto-x$ is continuous at 0 .

(5) $\forall \mu \epsilon \Sigma(0), \forall \delta \epsilon I_{0} \exists \nu \epsilon \Sigma(0) \ni \nu \odot \nu \leq \mu+\underline{\underline{\delta}}$, i.e., the mapping $(x, y) \mapsto x y$ is continuous at $(0,0)$.

\section{CHARACTERIZATION OF FNCDR AND SOME OTHER RESULTS.}

The following is a characterization of fuzzy neighborhood commutative division ring. We consider $\Sigma(0)$ to be symmetric fuzzy neighborhoods of zero.

THEOREM 3.1. Let $(D,+, \cdot)$ be a commutative division ring and $(D,+, \cdot t(\Sigma))$ a fuzzy neighborhood ring. Then the quadruple $(D,+, \cdot, t(\Sigma))$ is a fuzzy neighborhood commutative division ring if and only if the following are fulfilled:

(i) $\forall x \in D: \Sigma(x)=\left\{T_{x}(\nu)=x \oplus \nu ; \nu \in \Sigma(0)\right\}$.

(ii) $\forall \mu \epsilon \Sigma(0), \forall x \epsilon D, \forall \delta \epsilon I_{0} \exists \nu \epsilon \Sigma(0) \ni x \odot \nu \leq \mu+\underline{\underline{\delta}}$; i.e., $y \mapsto y x$ is continuous at 0 .

(iii) $\forall \mu \epsilon \Sigma(0), \forall \delta \epsilon I_{0} \exists \nu \epsilon \Sigma(0) \ni \nu \oplus \nu \leq \mu+\underline{\underline{\delta}}$, i.e., $(x, y) \mapsto x+y$ is continuous at $(0,0)$.

(iv) $\forall \mu \epsilon \Sigma(0), \forall \delta \epsilon I_{0} \exists \nu \epsilon \Sigma(0) \ni \nu \odot \nu \leq \mu+\underline{\underline{\delta}}$, i.e., $(x, y) \mapsto x y$ is continuous at $(0,0)$.

(v) $\forall \mu \epsilon \Sigma(0), \forall \delta \in I_{0} \exists \nu \in \Sigma(0) \ni(1 \oplus \nu) \sim \leq(1 \oplus \mu)+\underline{\underline{\delta}}$, i.e., the inversion $x \mapsto x^{-1}(x \neq 0)$ is continuous at 1 .

PROOF. If $(D,+, \cdot t(\Sigma))$ is a fuzzy neighborhood commutative division ring, then the conditions (i) - (iv) are immediate from Theorem 2.5. We check condition (v).

Let $\mu \in \Sigma(0)$ and $\delta \epsilon I_{0}$; then $1 \oplus \mu \epsilon \Sigma(r(1))$. Since $r: x \mapsto x-1$ is continuous at 1 , we can find $\nu \in \Sigma(0)$ such that $1 \oplus \nu \in \Sigma(1)$ and $r(1 \oplus \nu) \leq(1 \oplus \mu)+\underline{\underline{\delta}}$.

But $r(1 \oplus \nu) \leq(1 \oplus \nu) \sim$, so $(1 \oplus \nu) \sim \leq(1 \oplus \mu)+\underline{\underline{\delta}}$.

Conversely, if the conditions (i) - (v) are fulfilled then only we need to prove that the inversion $r: x \mapsto x^{-1}$ is continuous, i.e., we show that

$$
\begin{gathered}
\forall \mu \epsilon \Sigma(0), \forall x \epsilon D, \quad \forall \delta \in I_{0} \exists \nu \epsilon \Sigma(0) \ni \\
(x \oplus \nu)^{\sim} \leq(x \sim \oplus \mu)+\delta .
\end{gathered}
$$

Let $x \epsilon D^{*}, \mu \epsilon \Sigma(0)$ and $\delta \epsilon I_{0}$. Then in view of (ii), there is a $\mu_{1} \epsilon \Sigma(0)$ such that

$$
\mu_{1} \odot x \sim \underline{\mu}+\delta / \underline{\underline{3}}
$$

Now due to (v), corresponding to $\mu_{1}$ we can find $\nu_{1} \epsilon \Sigma(0)$ such that

$$
\left(1 \oplus \nu_{1}\right) \sim \leq\left(1 \oplus \mu_{1}\right)+\delta / \underline{\underline{3}} .
$$

Then by (ii), there exists a $\nu \epsilon \Sigma(0)$ such that

$$
(x \sim \odot \nu) \leq \nu_{1}+\delta / \underline{\underline{3}} .
$$

Now 


$$
\begin{aligned}
(1 \oplus(x \sim \odot \nu))^{\sim} & \leq\left(1 \oplus \nu_{1}\right) \sim+\delta / \underline{\underline{3}}(\text { from }(3.3)) \\
& \leq\left(1 \oplus \mu_{1}\right)+2 \delta / \underline{\underline{3}}(\text { from }(3.2)) \\
& \leq(1 \oplus(x \odot \mu))+(2 \delta / \underline{\underline{3}})+(\delta / \underline{\underline{3}})(\text { from }(3.1)) .
\end{aligned}
$$

But then with simplification, we have

$$
(x \oplus \nu) \sim=x \sim \odot(1 \oplus(x \sim \odot \nu))^{\sim} \leq(x \sim \oplus \mu)+\underline{\underline{\delta}} .
$$

which proves $(*)$.

PROPOSITION 3.2. Let $(D,+, \cdot, t(\Sigma))$ be a fuzzy neighborhood commutative division ring. If the conditions (i) - (v) of Theorem 3.1 are satisfied then the following inequality hold good.

$$
\forall \mu \epsilon \Sigma(0), \forall \delta \epsilon I_{0} \exists \nu \epsilon \Sigma(0) \ni \nu /(1 \oplus \nu) \leq \mu+\underline{\underline{\delta}} .
$$

PROOF. Suppose that the condition (i) - (v) hold good. Let $\mu \epsilon \Sigma(0)$ and $\delta \epsilon I_{0}$. Then there are $\mu_{1}, \mu_{2} \epsilon \Sigma(0)$ such that

$$
\mu_{1} \oplus \mu_{1} \leq \mu+\delta / \underline{\underline{3}} ; \mu_{2} \leq \mu_{1}
$$

and

$$
\mu_{2} \odot \mu_{2} \leq \mu_{1}+\delta / \underline{\underline{3}} .
$$

By (v), for every $\mu_{2} \epsilon \Sigma(0) \exists \nu \epsilon \Sigma(0), \nu \leq \mu_{2}$ such that

$$
(1 \oplus \nu)^{\sim} \leq\left(1 \oplus \mu_{2}\right)+\delta / \underline{\underline{3}} .
$$

Then we have

$$
\begin{aligned}
\nu /(1 \oplus \nu) & =\nu \odot(1 \oplus \nu) \sim(\text { by definition }) \\
& \left.\leq \nu \odot\left(1 \oplus \mu_{2}\right)+\delta / \underline{\underline{3}} \leq(\nu \odot 1) \oplus\left(\nu \odot \mu_{2}\right)+\delta / \underline{\underline{3}} \quad \text { by }(3.5)\right) \\
& \leq \mu_{2} \oplus\left(\mu_{2} \odot \mu_{2}\right)+\delta / \underline{\underline{3}} \\
& \leq\left(\mu_{2} \oplus \mu_{1}\right)+2 \delta / \underline{\underline{3}} \\
& \leq\left(\mu_{1} \oplus \mu_{1}\right)+2 \delta / \underline{\underline{3}} \\
& \leq \mu+\underline{\underline{\delta}} \\
& \Rightarrow \nu /(1 \oplus \nu) \leq \mu+\underline{\underline{\delta}} .
\end{aligned}
$$

THEOREM 3.3. Let $(D,+, \cdot)$ be a commutative division ring equipped with a fuzzy neighborhood topology $t(\Sigma)$. If $(D,+, t(\Sigma))$ is a fuzzy neighborhood group with respect to addition $h:(D \times D, t(\Sigma) \times t(\Sigma)) \rightarrow\left(D, t(\Sigma),(x, y) \mapsto x+y\right.$ and $\left(D^{*}, \cdot, t(\Sigma)\right)$ is a fuzzy neighborhood group with respect to multiplication $m:\left(D^{*} \times D^{*}, t(\Sigma) \times t(\Sigma)\right) \rightarrow\left(D^{*}, t(\Sigma)\right),(x, y) \mapsto x y$, then $(D,+, \cdot, t(\Sigma))$ is a fuzzy neighborhood commutative division ring.

PROOF. As the inversion, the addition and subtraction, i.e.,

$$
\begin{aligned}
& r:\left(D^{*}, t(\Sigma)\right) \rightarrow\left(D^{*}, t(\Sigma)\right), x \mapsto x^{-1}, \\
& h:(D \times D, t(\Sigma) \times t(\Sigma)) \rightarrow(D, t(\Sigma)),(x, y) \mapsto x+y ; \\
& h^{\prime}:(D \times D, t(\Sigma) \times t(\Sigma)) \rightarrow(D, t(\Sigma)),(x, y) \mapsto x-y
\end{aligned}
$$


are continuous, it is sufficient to show that the multiplication $m:(D \times D, t(\Sigma) \times t(\Sigma)) \rightarrow(D, t(\Sigma))$, $(x, y) \rightarrow x y$ is continuous.

Let $\Sigma(0)$ be symmetric fuzzy neighborhoods of zero in the additive group $D^{+}$of $D$, and

$$
\Sigma(x):=\{x \oplus \nu: \nu \epsilon \Sigma(0)\} \sim
$$

We show that

$$
\begin{aligned}
& \forall x \epsilon D, \forall y \epsilon D, \forall \mu \epsilon \Sigma(0), \forall \delta \epsilon I_{0} \exists \nu \epsilon \Sigma(0) \ni \\
& (\nu \oplus x) \odot(\nu \oplus y)-\underline{\underline{\delta}} \leq \mu \oplus x y .
\end{aligned}
$$

Condition (**) is satisfied for all $x \in D^{*}, y \epsilon D^{*}$.

Indeed,

$$
\begin{gathered}
\forall x, y \in D^{*}, \forall \mu_{x y} \epsilon \Sigma(x y), \forall \delta \epsilon I_{0} \exists \theta_{x} \epsilon \Sigma(x) \exists \theta_{y} \epsilon \Sigma(y) \ni \\
\theta_{x} \odot \theta_{y}-\underline{\delta} \leq \mu_{x y} .
\end{gathered}
$$

We let $\mu_{x y}:=\mu \oplus x y$ with $\mu \epsilon \Sigma(0)$,

$$
\theta_{x}:=x \oplus \theta \epsilon \Sigma(x) \theta_{y}:=y \oplus \theta^{\prime} \epsilon \Sigma(y) \text { with } \theta, \theta^{\prime} \epsilon \Sigma(0) \text {. }
$$

Put $\nu:=\theta \wedge \theta^{\prime}$. Then we have

$$
\begin{aligned}
(\nu \oplus x) \odot(\nu \oplus y)-\underline{\underline{\delta}} & \leq(\theta \oplus x) \odot\left(\theta^{\prime} \oplus x\right)-\underline{\underline{\delta}} \\
& \leq \theta_{x} \odot \theta_{y}-\underline{\underline{\delta}} \leq \mu_{x y}=\mu \oplus x y
\end{aligned}
$$

as desired.

It remains to show that if $x y=0$, then $(* *)$ is satisfied. First, let $x=y=0$; suppose $\mu \epsilon \Sigma(0)$ and $\delta \epsilon I_{0}$; then by Proposition 2.3(c), $1 \oplus \mu \epsilon \Sigma(1)$.

There exists $\theta \epsilon \Sigma(0)$ such that

$$
\theta \oplus \theta \oplus \theta \leq \mu+\delta / \underline{\underline{2}}
$$

Consequently, as multiplication $m:(x, y) \rightarrow x y$ is continuous at $(1,1)$, there exists $\nu_{1} \epsilon \Sigma(1)$ such that

$$
\nu_{1} \odot \nu_{1} \leq(1 \oplus \theta)+\delta / \underline{\underline{2}} .
$$

Then in view of Proposition 2.3(d), $-1 \oplus \nu_{1} \epsilon \Sigma(0)$. Let us put

$$
\nu:=-1 \oplus \nu_{1} \text { and } \nu:=\nu \wedge \theta
$$

then $\nu \in \Sigma(0)$ and hence,

$$
\begin{aligned}
\nu \odot \nu & =\left(-1 \oplus \nu_{1}\right) \odot\left(-1 \oplus \nu_{1}\right) \\
& \leq 1 \oplus\left((-1) \odot \nu_{1}\right) \oplus\left(\nu_{1} \odot(-1)\right) \oplus\left(\nu_{1} \odot \nu_{1}\right) \\
& \leq\left(1 \oplus\left(\sim \nu_{1}\right)\right) \oplus\left(\sim \nu_{1}\right) \oplus(1 \oplus \theta)+\delta / \underline{\underline{2}} \\
& \leq \theta \oplus \theta \oplus \theta+\delta / \underline{\underline{2}} \leq \mu+\underline{\underline{\delta}}
\end{aligned}
$$

which proves that

$$
\forall \mu \epsilon \Sigma(0), \forall \delta \epsilon I_{0} \exists \nu \epsilon \Sigma(0) \ni \nu \odot \nu \leq \mu+\delta .
$$


Next, let $x \neq 0=y$. Since the multiplication $m:(x, y) \mapsto x y$ is continuous at $(1, x)$, then $(* * *)$ implies that

$$
\forall \mu_{x} \epsilon \Sigma(x), \forall \delta \epsilon I_{0} \exists \nu_{1} \epsilon(1) \exists \nu_{x} \epsilon \Sigma(x) \ni \nu_{1} \sigma \nu_{x}-\underline{\underline{\delta}} \leq \mu_{x}
$$

Choose

$$
\begin{gathered}
\mu_{x}:=\mu \oplus x, \text { with } \mu \in \Sigma(0) ; \\
\nu_{1}:=1 \oplus \theta, \nu_{x}:=x \oplus \theta^{\prime} \text { with } \theta, \theta^{\prime} \in \Sigma(0) .
\end{gathered}
$$

Set $\nu:=\theta \wedge \theta^{\prime}$. Then it follows immediately that

$$
(1 \oplus \nu) \odot(x \oplus \nu) \leq(\mu \oplus x)+\underline{\underline{\delta}} .
$$

(by (3.8))

and consequently, $(\nu \oplus x) \odot \nu \leq \mu+\underline{\underline{\delta}}$ which proves that

$$
\forall \mu \epsilon \Sigma(0), \forall x \epsilon D^{*}, \forall \delta \epsilon I_{0} \exists \nu \epsilon \Sigma(0) \ni(\nu \oplus x) \odot \nu \leq \mu+\underline{\underline{\delta}} .
$$

DEFINITION 3.4. Let $(D,+, \cdot)$ be a commutative division ring and $(D,+, \cdot, t(\Sigma))$ a fuzzy neighborhood ring. Then a fuzzy set $\mu \epsilon I^{D}$ is said to be bounded in $(D,+, \cdot t(\Sigma))$ if and only if for all $u \in \Sigma(0)$ and for all $\delta \epsilon I_{0}$ there exists $\theta \epsilon \Sigma(0)$ such that $\mu \odot \theta \leq \nu+\underline{\underline{\delta}}$.

PROPOSITION 3.5. Let $(D,+, \cdot)$ be a commutative division ring and $(D,+, \cdot t(\Sigma))$ a fuzzy neighborhood ring. Then the following statements are equivalent:

(B1): $\mu \in I^{D}$ is bounded in $(D,+, \cdot, t(\Sigma))$;

(B2): $\forall \nu \epsilon \Sigma(0), \forall \delta \epsilon I_{0} \exists x \epsilon D^{*} \ni \mu \odot x \leq \nu+\underline{\underline{\delta}}$.

PROOF. $(\mathrm{B} 1) \Rightarrow(\mathrm{B} 2)$ is trivial, we prove $(\mathrm{B} 2) \Rightarrow(\mathrm{B} 1)$. Let $\mu \epsilon I^{D}, \nu \in \Sigma(0)$ and $\delta \in I_{0}$. Then in view of Theorem 3.1 (iv) there exists a $\nu^{\prime} \in \Sigma(0)$ such that

$$
\nu^{\prime} \odot \nu^{\prime}-\delta / \underline{\underline{3}} \leq \nu
$$

By hypothesis, there is $x \in D^{*}$ such that

$$
\boldsymbol{\mu} \odot \boldsymbol{x}-\delta / \underline{\underline{3}} \leq \nu^{\prime}
$$

Thus we have

$$
\begin{gathered}
\nu^{\prime} \odot(\mu \odot x) \underset{(b y(3.10))}{\leq} \nu^{\prime} \odot \nu^{\prime}+\delta / \underline{\underline{3}} \\
\underset{(b y)(3.9))}{\leq} \tilde{\nu}+2 \delta / \underline{\underline{3}}
\end{gathered}
$$

Again applying Theorem 3.1(ii), we can find $\theta \epsilon \Sigma(0)$ such that

So for any $z \in D$ :

$$
\begin{aligned}
& \theta \odot x \sim \leq \nu^{\prime}+\delta / \underline{\underline{3}} \\
& \Rightarrow \theta \leq \nu^{\prime} \odot x+\delta / \underline{\underline{3}}
\end{aligned}
$$

$$
\begin{aligned}
& \mu \odot \theta(z) \quad=\quad \stackrel{\vee}{\vee}=z^{\mu} \mu(s) \wedge \theta(t) \\
& \leq \quad \stackrel{v}{=} z^{\mu}(s) \wedge\left(\nu^{\prime} \odot x\right)(t)+\delta / 3 \\
& =\mu \odot\left(\nu^{\prime} \odot x\right)(z)+\delta / 3 \\
& \leq \quad \nu(z)+2 \delta / 3+\delta / 3=\nu(z)+\delta \\
& \text { (by(3.11)) } \\
& \Rightarrow \mu \odot \theta \quad \leq \quad \nu+\underline{\underline{\delta}} .
\end{aligned}
$$


DEFINITION 3.6. Let $(D,+, \cdot)$ be a commutative division ring and $(D,+, \cdot, t(\Sigma))$ a fuzzy neighborhood ring. A fuzzy set $\mu \in I^{D}$ is said to be $\beta$-restricted in $(D,+, \cdot, t(\Sigma))$ for $0<\beta \leq 1$ if and only if

$$
\overline{\mu^{\sim}}(0)<\beta,
$$

Where ${ }^{-}$is the fuzzy closure operator given in Proposition 2.3 [6]

PROPOSITION 3.7. Let $(D,+, \cdot)$ be a division ring and $(D,+, \cdot t(\Sigma))$ a fuzzy neighborhood ring. Then the following statements are equivalent:

(R1): $\mu \epsilon I^{D}$ is $\beta$-restricted in $(D,+, \cdot, t(\Sigma))$ for $0<\beta \leq 1$;

(R2): $\exists \nu \epsilon \Sigma(0) \ni \mu \odot \nu(1)<\beta$.

PROOF. $\cdot(\mathrm{R} 1) \Rightarrow(\mathrm{R} 2)$. Let $0<\beta \leq 1$, and $\mu \epsilon I^{D}$ be $\beta$-restricted. Suppose that $\nu \epsilon \Sigma(0)$ is such that $\mu \odot \nu(1) \geq \beta$; i.e., $\vee_{x y=1} \mu(x) \wedge \nu(y) \geq \beta$

$\Rightarrow \exists x \in D, y \in D^{*}$ such that $x y=1$, i.e., $x=y^{-1}$ such that

$$
\begin{gathered}
\mu\left(y^{-1}\right) \wedge \nu(y) \geq \beta \\
\Rightarrow \underset{\nu \in \Sigma(0)}{\underset{y \in D^{*}}{\vee} \mu\left(y^{-1}\right) \wedge \nu(y) \geq \beta} \\
\Rightarrow \underset{\nu \in \Sigma(0)}{\underset{y \in D^{*}}{\vee} \mu \sim(y) \wedge \nu(y) \geq \beta}
\end{gathered}
$$

$\Rightarrow \bar{\mu}(0) \geq \beta$, contradiction with the fact that $\mu$ is $\beta$-restricted. (R2) $\Rightarrow(\mathrm{R} 1)$. Let $\mu \in I^{D}$ be not $\beta$ restricted for $0<\beta \leq 1$.

This means simply that

Now we have

$$
\begin{gathered}
\underset{\nu \in \Sigma(0)}{\underset{\nu \in D^{*}}{\vee} \mu^{\sim} \sim(y) \wedge \nu(y) \geq \beta} \\
\Rightarrow \forall \nu \in \Sigma(0): \underset{y \in D^{*}}{\vee} \mu^{\sim}(y) \wedge \nu(y) \geq \beta .
\end{gathered}
$$

$$
\begin{aligned}
\mu \odot \nu(1) & =\underset{t t=1}{\vee} \mu(s) \wedge \nu(t) \\
& =\underset{t=t^{-1} \in D^{*}}{\vee} \mu^{\sim}(t) \wedge \nu(t) \geq \beta
\end{aligned}
$$

a contradiction with (R2).

\section{REFERENCES}

1. AHSANULLAH, T.M.G., Fuzzy neighborhood modules and algebra, Fuzzy Sets and Systems 35, (1990), 219-229.

2. AHSANULLAH, T.M.G. \& GANGULY, S., Fuzzy neighborhood rings, Fuzzy Sets and Systems 34, (1990), 255-262.

3. AHSANULLAH, T.M.G. \& MORSI, N.N., Invariant probabilistic metrizability of fuzzy neighborhood groups, Fuzzy Sets and Systems 47, (1992), 233-245.

4. KATSARAS, A.K., Linear fuzzy neighborhood spaces, Fuzzy Sets and Systems 16, (1985), 25-40.

5. MORSI, N.N., Nearness concepts in fuzzy neighborhood spaces and in their fuzzy proximity spaces, Fuzzy Sets and Systems 31, (1989), 83-109. 
6. LOWEN, R., Fuzzy neighborhood spaces, Fuzzy Sets and Systems 7, (1982), 165-189.

7. LOWEN, E., LOWEN, R. \& WUYTS, P., The categorical topology approach to fuzzy topology and fuzzy convergence, Fuzzy Sets and Systems 40, (1991), 347-373.

8. KAPLANSKY, I., Topological rings, Amer. J. Math. 69, (1947), 153-182.

9. WANG-JIN, L., Operations on fuzzy ideals, Fuzzy Sets and Systems 11, (1983), 31-41. 


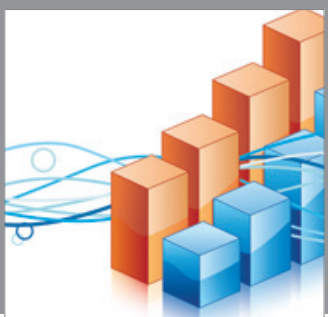

Advances in

Operations Research

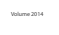

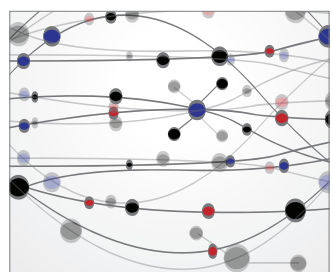

\section{The Scientific} World Journal
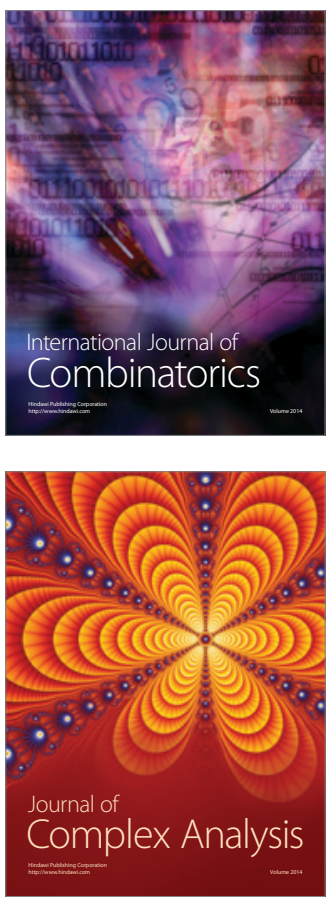

International Journal of

Mathematics and

Mathematical

Sciences
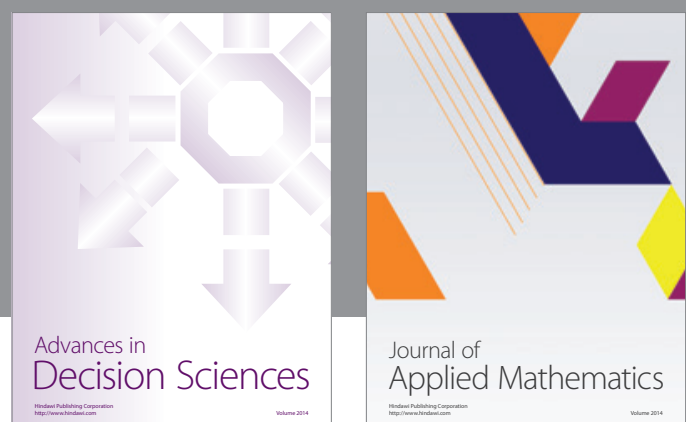

Journal of

Applied Mathematics
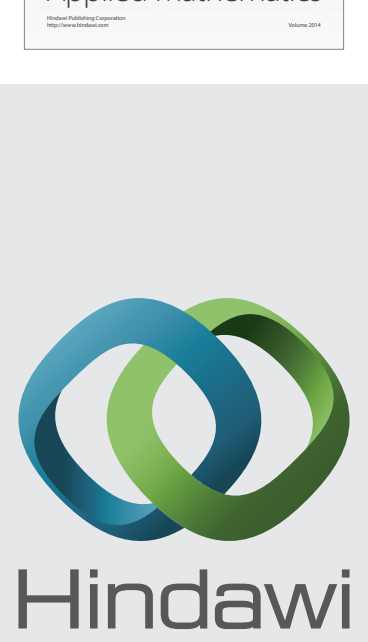

Submit your manuscripts at http://www.hindawi.com
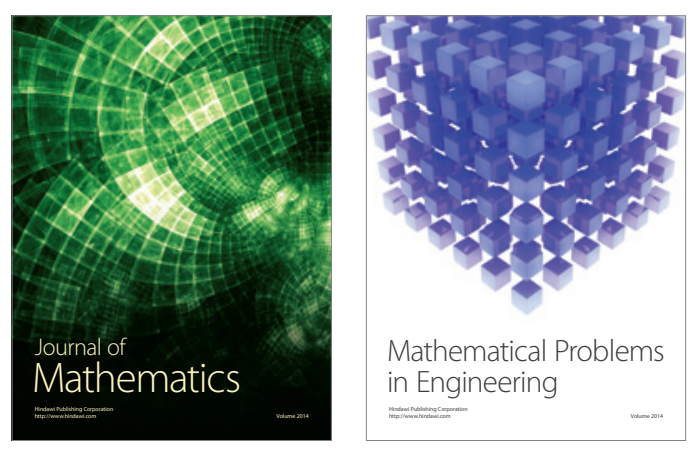

Mathematical Problems in Engineering
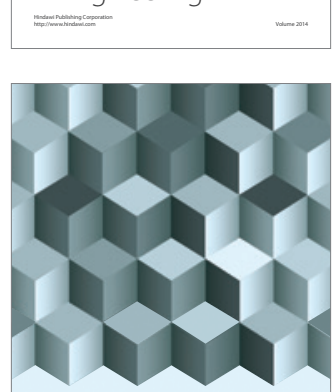

Journal of

Function Spaces
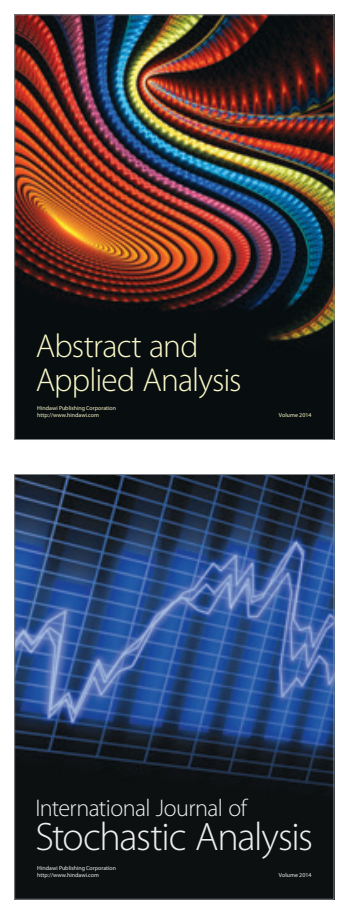

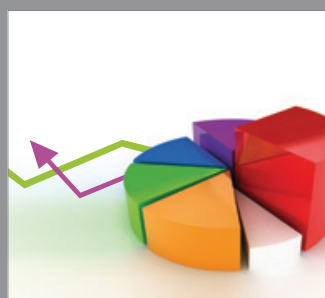

ournal of

Probability and Statistics

Promensencen
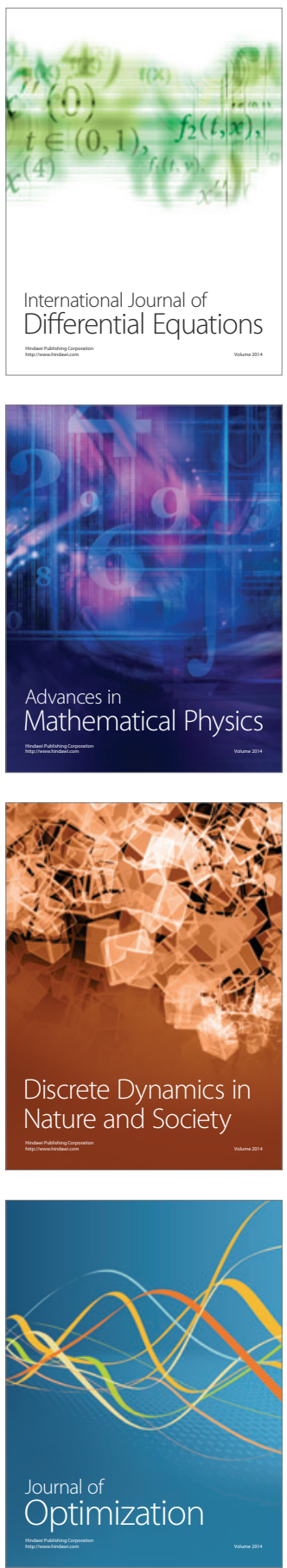